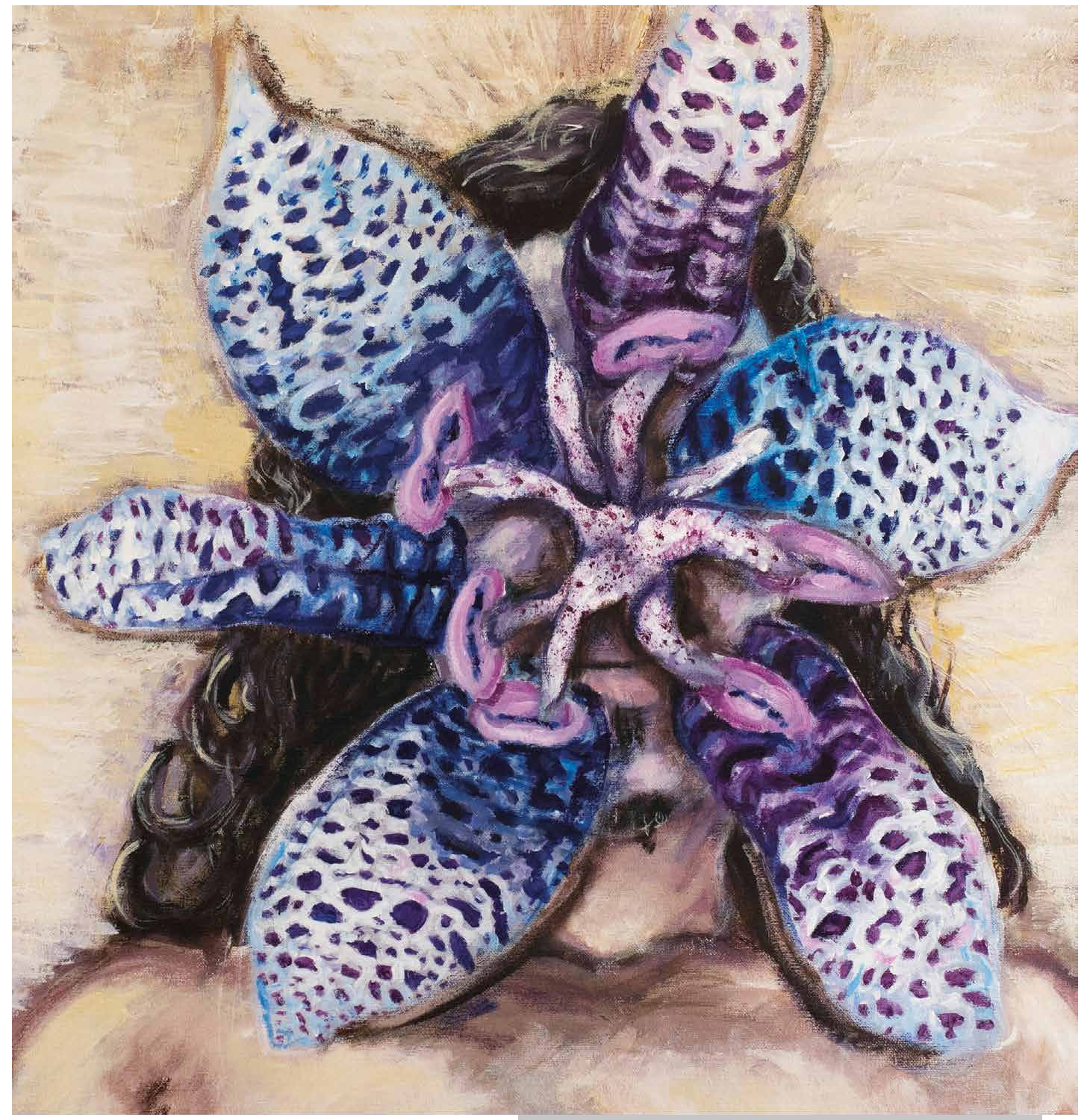

Artista invitado

Rafael Germán Rengifo Sánchez

La sonrisa de la melancolía

De la serie El jardín detrás de mi cabeza

Acrílico sobre lona

$60 \times 50 \mathrm{~cm}$

2020

Medellín 


\title{
Burócratas y ciudadanos. La burocracia de nivel de calle en la implementación de la política pública de seguridad alimentaria y nutricional en el departamento de Antioquia*
}

\author{
Germán Ignacio Casas Arango (Colombia)** \\ Cristian Daniel Aguirre Henao (Colombia)*** \\ Lorena Patricia Mancilla López (Colombia) ${ }^{* * * *}$
}

\section{Resumen}

Los burócratas de nivel de calle son aquellos funcionarios que tienen una interacción directa con los ciudadanos para la prestación y entrega de bienes y servicios, por lo que sus acciones al momento de implementar las políticas resultan determinantes. A partir de un análisis cualitativo de 193 entrevistas se evidencia el papel que desempeñan los burócratas de nivel de calle en la política pública de seguridad alimentaria y nutricional en el departamento de Antioquia, Colombia. Estos burócratas, que hacen uso de su discrecionalidad, producen cambios en los lineamientos de las políticas con el fin de incluir a beneficiarios que consideren más adecuados según los fines de la política. Asimismo, realizan acciones adicionales que van más allá de las funciones asignadas para dar un acompañamiento más integral a los beneficiarios. Estos burócratas adoptan diferentes conductas según el tipo de ciudadano al que se le provee el bien o servicio, derivando en una forma particular de la política pública en cuestión.

\footnotetext{
* Artículo derivado del trabajo de grado homónimo para optar al título de magíster en Políticas Públicas, Universidad de Antioquia, Colombia, bajo la dirección de Juan Pablo Mesa Mejía y Lorena Patricia Mancilla. Este también es resultado del componente de análisis de redes sociales y mapeo de actores del Perfil Alimentario y Nutricional de Antioquia (PANA) 2019, desarrollado por la Gobernación de Antioquia en convenio con la Universidad de Antioquia.

** Economista y abogado. Magíster en Políticas Públicas. Profesor Facultad de Ciencias Económicas, Universidad de Antioquia UdeA. Calle 70 No. 52-21, Medellín, Colombia. Correo electrónico: german. casas@udea.edu.co - Orcid: 0000-0001-8125-3920

*** Politólogo. Magíster en Políticas Públicas. Profesor Facultad de Derecho y Ciencias Políticas, Universidad de Antioquia UdeA. Calle 70 No. 52-21, Medellín, Colombia. Correo electrónico: cdaniel. aguirre@udea.edu.co - Orcid: 0000-0003-3871-1089 - Google Scholar: https://scholar.google.com.co/ citations?user $=6$ sbiPq4AAAAJ\&hl $=$ es

${ }^{* * * *}$ Nutricionista-dietista. Magíster en Ciencias Ambientales. Doctora en Salud Pública. Profesora Escuela de Nutrición y Dietética, Universidad de Antioquia UdeA. Calle 70 No. 52-21, Medellín, Colombia. Correo electrónico: lorena.mancilla@udea.edu.co - Orcid: 0000-0001-8090-8497 - Google Scholar: https://scholar.google.com/citations?hl=es\&user=6sbiPq4AAAAJ
} 


\title{
Palabras clave
}

Políticas Públicas; Implementación de Políticas; Burócratas de Nivel de Calle; Seguridad Alimentaria y Nutricional; Antioquia, Colombia.

Fecha de recepción: julio de 2020

- Fecha de aprobación: noviembre de 2020

\section{Cómo citar este artículo}

Casas, Germán Ignacio; Aguirre Henao, Cristian Daniel y Mancilla, Lorena Patricia. (2021). Burócratas y ciudadanos. La burocracia de nivel de calle en la implementación de la política pública de seguridad alimentaria y nutricional en el departamento de Antioquia. Estudios Políticos (Universidad de Antioquia), 61, pp. 125-152. DOI: 10.17533/udea.espo.n61a06

\section{Bureaucrats and Citizens. Street-level Bureaucracy in the Implementation of Public Policy on Food and Nutritional Security in the Department of Antioquia}

\begin{abstract}
[126] Street-level bureaucrats, such as officials who have direct interaction with citizens for the provision and delivery of goods and services, play an important role when implementing public policies. Based on a qualitative analysis of 193 interviews, it was evinced the role that street-level bureaucrats play in the public food and nutrition security policy of the Department of Antioquia, Colombia. These bureaucrats, making use of their own discretion, produce changes in the policy parameters for the inclusion of beneficiaries that they consider more suitable according to the purposes of the policy. Likewise, they carry out actions that go beyond the assigned functions in order to provide a more comprehensive support to the beneficiaries. These bureaucrats adopt different behaviors depending on the type of citizen to whom the good or service is provided, resulting in a particular form of implementation of the public policy in question.
\end{abstract}

\section{Keywords}

Public Policies; Policies Implementation; Street-Level Bureaucrats; Food and Nutrition Security; Antioquia, Colombia. 


\section{Introducción}

La implementación de políticas públicas se puede concebir como «el proceso de convertir un mero enunciado mental (legislación, plan o programa de gobierno) en un curso de acción efectivo, y es el proceso de convertir algo que es solo un deseo, un efecto probable, en una realidad efectiva» (Aguilar, 1992, p. 13). Este proceso de implementación, en el que se pasa del dicho al hecho, tiene cuatro grandes retos denominados «grupos generales de influencia» (Hill, 2018, p. 130), en los cuales se enmarcan, a su vez, un sinnúmero de variables que pueden afectar el proceso de implementación de la política. El primer reto hace referencia a «la política pública y el proceso de las políticas», cuyos aspectos que deben acompañar el diseño de la política en sí son los que moldean los resultados, tales como los recursos asignados, la adecuada identificación del problema público, el establecimiento de una teoría causal sólida y la presencia de actores responsables de solucionar el problema en cuestión. El segundo reto se encuentra asociado a «las organizaciones y su medio», puesto que las características organizacionales configuran la política como resultado de la acción gubernamental. El tercer reto está asociado a «los agentes», en el que las preferencias y capacidades de los actores involucrados en el proceso de la política pueden contribuir a darle forma. Finalmente, el cuarto reto se relaciona con «las condiciones dentro del entorno de implementación», en el cual se pueden encontrar variables como las condiciones económicas, el comportamiento del grupo objetivo, los beneficiarios y grupos de interés.

A su vez, estos retos pueden asociarse al implementation gap, el cual expresa la disonancia entre lo formulado y lo implementado. Estas brechas dan cuenta de las diferencias existentes entre lo diseñado y establecido por los tomadores de decisiones y lo que realizan los funcionarios y contratistas que las ejecutan. Por ello, la implementación como parte del proceso de la política pública se define como el momento más álgido y determinante, y las fallas y dificultades que se presentan condicionan el cumplimiento de sus objetivos. Estas brechas son producto de factores como:

- El alto nivel burocrático, lo que reduce la rapidez y flexibilidad de intervención (Kaufman, 1973; Whitford, 2002) al exacerbar los controles y procesos, generando incentivos negativos para los actores al emprender nuevas acciones que mejoren la implementación de las políticas (Espinoza y Huita, 2012). 
— La falta de recontextualización (Medellín, 2004) y territorialización de las políticas (Jolly, 2007), lo cual genera una inadecuada adaptación al momento de intervenir en la realidad particular del lugar donde se realizará la acción, dificultando la implementación de la política.

- La desinformación al momento de tomar decisiones y la ambigüedad en el diseño de políticas (Zahariadis, 2010), lo que genera vaguedad, confusión y tensión, repercutiendo en el proceso de implementación.

- La alta complejidad del factor político, dado que los actores - públicos y privados - involucrados en el proceso de implementación de la política pública pueden modificar las reglas de juego y orientarlas hacia sus intereses particulares mediante sus visiones y valores (Pressman y Wildavsky 1984; Knoepfel, Larrue, Varone e Hinojosa, 2007).

Así, la presencia de estas brechas se asocia, en gran medida, a que quienes diseñan y toman las decisiones no son quienes implementan las políticas públicas, las cuales son el resultado de una cadena de relaciones entre formuladores e implementadores ubicados en diferentes ámbitos de gobierno. Es así como la proximidad entre las intenciones del formulador y [128 ] la ejecución de las políticas influye de manera directa en su efecto (Arretche, 2001, p. 49). Por esto, su entendimiento puede transformarse a través de la cadena de mando hasta llegar a quienes tienen contacto directo con los ciudadanos y se encargan de proveer bienes y servicios para dar solución a sus demandas y problemáticas particulares. Por lo anterior, cabe resaltar que los actores que diseñan e implementan las políticas no son neutrales ni pasivos, pues el conflicto es parte inherente de su interacción, «particularmente a raíz de los niveles de discrecionalidad de los que goza cada uno a la hora de implementar la parte del programa que les compete» (Navarrete y Figueroa, 2019, p. 101).

Es así como los funcionarios y contratistas de primera línea, también llamados burócratas de nivel de calle (BNC), o street-level bureaucracy en la literatura académica, dejan de ser vistos como los últimos eslabones de la cadena de poder para entrar a desempeñar un papel determinante en el éxito o fracaso de la intervención gubernamental. Los BNC deben enfrentar los errores y complicaciones que se presenten, siguiendo un marco normativo y procedimental que no siempre se ajusta o logra responder a las particularidades de cada caso. Esto implica que deben decidir entre seguir las 
reglas preestablecidas o modificarlas con el fin de dar solución aparente a las necesidades de cada ciudadano (Lipsky, 2010).

Desde esta perspectiva se busca entender cómo los BNC configuran la Política Pública de Seguridad Alimentaria y Nutricional (PPSAN) del departamento de Antioquia, Colombia, aprobada a través de la ordenanza 046 de 2016. Este documento de política pública establece, entre otras cosas, la disponibilidad y acceso, el consumo y aprovechamiento biológico, y la calidad e inocuidad de los alimentos como componentes estratégicos y transversales, bajo los cuales se desarrollan acciones como proyectos productivos como huertas, asistencia técnica agropecuaria, complementación alimentaria a través del Programa de Alimentación Escolar (PAE) y paquetes alimentarios, recuperación nutricional, atención integral nutricional a través de Centros de Desarrollo Infantil (CDI), entre otros programas y proyectos que se condensan en el Plan Docenal de Seguridad Alimentaria y Nutricional del departamento de Antioquia y los planes de desarrollo municipales y departamentales.

En este marco, se busca analizar cómo la discrecionalidad y autonomía de los BNC, en consonancia con factores territoriales e institucionales como la normatividad, la capacidad técnica, política y económica, y su relación con la ciudadanía pueden generar una reformulación de facto de la política que se distancie de los lineamientos provistos por los documentos, excediendo sus propósitos o reduciéndose al quehacer particular de la organización y sus funcionarios.

\section{La burocracia en los estudios de implementación de políticas públicas}

El concepto de burocracia ha variado considerablemente durante los últimos años. Con los aportes realizados por Max Weber (1993) se definió a la burocracia como el conjunto de actores racionales que se rigen por las normas, tienen una clara jerarquía de mando, rutinas y procedimientos preestablecidos y son capaces de garantizar el cumplimiento de objetivos. Posteriormente, Henry Mintzberg (1984) denominó esto como «burocracia maquinal» para describir al conjunto de individuos organizados jerárquicamente que cumplen funciones rutinarias con una estructura de trabajo fija y normalizada. Desde esta perspectiva, la burocracia en el proceso de implementación de las acciones de gobierno cuenta con una ventaja que deriva de su precisión, claridad, subordinación y permanencia en el tiempo (Mayntz, 1987). Esto 
permitiría actuar de manera objetiva en el marco de procesos formales con el fin de cumplir los objetivos establecidos.

Más adelante, Charles Wright Mills (1993) sentó una posición contraria a la weberiana, al considerar que las burocracias no son más que una fachada para conservar la sensación de racionalidad política en la toma de decisiones por parte de un grupo específico de individuos —élites- que van en pro de intereses particulares. Aunque esta postura fue fuertemente cuestionada, posicionó un discurso en el cual las burocracias carecen de objetividad y no son más que un mero requerimiento instrumental para alcanzar fines fijados por élites que determinan la vida política, económica y social de los territorios. Ambas posturas fueron fundamentales para explicar la diversidad de dinámicas políticas y los resultados del accionar gubernamental. Sin embargo, Michael Lipsky (1969) abrió una nueva puerta a esta discusión. Centró su atención en los burócratas de primera línea que tienen contacto directo con los ciudadanos en el marco de la provisión de bienes y servicios públicos, y que además se encuentran dotados de autonomía y discrecionalidad para aplicar las normas y procedimientos preestablecidos para la atención de la ciudadanía.

[130] El comportamiento de los BNC varía considerablemente entre políticas públicas. En ocasiones, estos pueden ceñirse a las reglas y procedimientos predefinidos, reducir su discrecionalidad voluntariamente y llevar a cabo de manera homogénea la intervención gubernamental. Sin embargo, también pueden hacer uso de su discrecionalidad y autonomía, flexibilizar los procedimientos y atender cada situación de manera particular, reconociendo así la heterogeneidad del contexto y de las necesidades de los ciudadanos. El cumplimiento de los objetivos propuestos depende de cada caso particular.

Los postulados de Lipsky (2010) y los aportes y críticas de diversos colaboradores han permitido establecer, al menos, cuatro grandes premisas teóricas que han sido ampliamente abordadas en múltiples estudios de casos empíricos en Europa y Norteamérica, permitiendo comprobar su validez y potencial explicativo. La primera se encuentra relacionada con la discrecionalidad que representa a los burócratas de nivel de calle, que a su vez tiene cuatro características: a) es inevitable, b) es constante, c) es inherente a los actos de la administración y d) está fuera del control de los supervisores - funcionarios o agencias gubernamentales- (Maynard-Moody y Musheno, 2018). 
Sin embargo, el punto nodal no es en sí la discrecionalidad, sino el posible uso de esta. Marcia Mayers, Bonnie Glaser y Karin MacDonald (1998, citados en Maynard-Moody y Musheno, 2018) calculan que «aproximadamente en el $75 \%$ de las entrevistas de admisión a beneficiarios de prestación de servicios sociales, los BNC actuaron sin desviarse del guion convencional». Esto implica que, en general, estos burócratas se rigen por las reglas y procedimientos preestablecidos para dar tratamiento a los ciudadanos-beneficiarios de programas sociales. No obstante, existe una evidente posibilidad de que estos hagan uso de su discrecionalidad para dar tratamiento a casos particulares que sobrepasan estos lineamientos o bien para beneficiar o sancionar a los ciudadanos en las situaciones que consideren necesarias.

La segunda premisa plantea que los BNC «son importantes tomadores de decisiones, quienes (re)formulan las políticas públicas de acuerdo con sus juicios y opiniones» (Dussage, Cejudo y Pardo, 2018, p. 20). Las decisiones que toman los BNC al momento de seguir los procedimientos dictaminados por los ámbitos superiores de mando tienen como consecuencia una configuración particular de las políticas públicas en el ámbito concreto en donde suceden las interacciones con los ciudadanos.

Cabe destacar que, en general, las políticas públicas buscan homogeneizar grandes grupos poblacionales bajo unas mismas condiciones de selección o participación. Esto hace que en casos particulares y excepcionales se haga uso de la discrecionalidad inherente a los BNC, aumentando así la probabilidad de generar impacto en los beneficiarios. Esto significa que, en ocasiones, los BNC hacen uso de su capacidad de juicio, la cual «les permite tratar al público de manera justa, adecuada y cuidadosa». En otros casos, su discrecionalidad puede derivar en la violación de la norma, cayendo así en el «favoritismo, los estereotipos, la comodidad y las conductas rutinarias, todo lo cual resulta útil para sus propios fines o los de la agencia» (Lipsky, 2018, p. 31).

La tercera premisa está relacionada con las bajas probabilidades que tienen los superiores de controlar la discrecionalidad y la toma de decisiones de los BNC. Pese a que «en general se veía al personal de primera línea como carente de poder» (Maynard-Moody y Musheno, 2018, p 42), las investigaciones han evidenciado su capacidad de incidencia en su ámbito de poder para configurar y desarrollar las políticas públicas. 
La cuarta y última premisa se relaciona con la amplitud y ambigüedad que acompaña a las políticas públicas, lo que genera falta de claridad para que los BNC lleven a cabo sus acciones (Hill, 2018). En este sentido, Heather C. Hill (2018) analiza el caso de Ley de Control de Crímenes Violentos y Orden Público en Estados Unidos, y afirma que la ausencia de pautas claras para que los BNC implementen las políticas públicas puede limitar sus resultados. En la misma línea, André-Noël Roth (2018) explica que esta ambigüedad se presenta porque muchas políticas públicas «son el resultado de negociaciones, de ajustes entre contradicciones y oposiciones, deseadas o no, que buscan satisfacer diversos intereses y valores» (pp. 200-201).

A la falta de concreción se le suma la ausencia de significados compartidos entre formuladores-hacedores y BNC con respecto a la política pública (Hill, 2018), lo que en ocasiones resulta en un mayor uso de la discrecionalidad para poder sortear la carencia de lineamientos claros y atender a los usuarios de las acciones de gobierno. Desde esta perspectiva, la discrecionalidad se hace no solo necesaria, sino también deseable, pues se presenta como solución a las decisiones desfavorables (Weatherley y Lipsky, 1977).

Bajo estas cuatro premisas, los BNC pueden considerarse como [132] Ios elaboradores finales y los actores más influyentes en el proceso de la política pública (Lipsky, 2010), cuyo actuar difícilmente puede controlarse en su totalidad.

Finalmente, cabe resaltar algunos aspectos frente a las limitaciones del trabajo a nivel calle (Lipsky, 2010; Hupe, 2019; Maynard-Moody y Portillo, 2018; Cohen y Klenk, 2019; Hill, 2018). En primer lugar, como se explicó anteriormente, la discrecionalidad no es positiva o negativa, sino que está sujeta al uso que el BNC haga de ella. En segundo lugar, llevar las políticas públicas a los ciudadanos implica una delegación de poder a sujetos autónomos con intereses, metas y formas de pensar particulares que se pueden entrelazar con los objetivos propios de las políticas, llevando a su transformación. En tercer lugar, los BNC se enfrentan cotidianamente a retos y limitaciones que pueden estar circunscritas a la política en sí, como la ambigüedad, amplitud o restricciones normativas, además de los factores políticos, económicos o sociales particulares del territorio donde se implementa, Ilevándolos a adaptarse y crear estrategias para sortear estas dificultades. Finalmente, en coherencia con lo anterior, los BNC hacen lo que pueden con los recursos políticos, económicos y jurídicos 
que estén a su disposición, ciñéndose a los lineamientos preestablecidos, sin dejar de lado su discrecionalidad cuando la requieran. Además, hacen lo que deben, aplican al pie de la letra los lineamientos de las políticas sin detenerse a pensar en los beneficios o perjuicios que pueden causarle a los ciudadanos, y hacen lo que quieren aprovechándose de la ambigüedad y la amplitud de las políticas, cayendo en el favoritismo y los estereotipos, y creando un «cliente ideal» que recibirá los bienes o servicios de la política pública.

\section{Marco metodológico}

La investigación se realizó con información obtenida del Perfil Alimentario y Nutricional de Antioquia (PANA), específicamente del Componente de Análisis de Redes Sociales y Mapeo de Actores que desarrolló la Universidad de Antioquia en conjunto con la Gobernación de Antioquia durante 2018 y 2019. De allí se tomaron 193 entrevistas semiestructuradas, 96 de hombres y 97 de mujeres, realizadas a ciudadanos y burócratas de diferentes ámbitos. Estas fueron llevadas a cabo por un equipo de investigación en las diferentes subregiones del departamento de Antioquia y se grabaron para su posterior transcripción.

Para analizar estas entrevistas se elaboró un diseño metodológico basado en el estudio de casos, además se emplearon los postulados de la teoría fundamentada para orientar la observación y codificación de los datos; asimismo, se tomaron como referentes las investigaciones de Tony Evans (2011), Anat Gofen (2013), Rod Williams (2014), Mikael Sevä (2015) y Shaveta Menon (2018), quienes emplean las entrevistas como principal instrumento para el desarrollo de sus investigaciones en materia de $\mathrm{BCN}$, realizando un análisis a profundidad de la información obtenida para cumplir los objetivos de la investigación.

El primer paso fue diferenciar a los entrevistados con el fin de definir los tipos de burócratas y distinguir a los BNC de otros técnicos y tomadores de decisiones, además de ubicar los ciudadanos partícipes del estudio. Cabe resaltar que allí se encontraron secretarios, subsecretarios, líderes de programas, coordinadores de proyectos, técnicos, ciudadanos y un significativo grupo de burócratas de nivel de calle a cargo de proyectos como huertas caseras, proyectos productivos, comedores sociales, comunitarios o escolares, paquetes alimentarios, entre otros propios de las políticas de 
seguridad alimentaria y nutricional de nivel territorial que se inscriben en el Plan Docenal de Seguridad Alimentaria y Nutricional 2020-2031 y en los planes de desarrollo municipales y se implementan conforme a ellos (MANÁ, 2020). En total, el estudio contó con 126 entrevistas realizadas a burócratas de diferentes ámbitos y 67 realizadas a ciudadanos (véase tabla 1).

Tabla 1. Procedencia, tipo y cantidad de entrevistados relacionados con la política de alimentación y nutrición en el departamento de Antioquia, 2018-2019.

\begin{tabular}{|c|c|c|c|c|}
\hline Municipio & Subregión & $\begin{array}{c}\text { Burócratas } \\
\text { entrevistados }\end{array}$ & $\begin{array}{l}\text { Ciudadanos } \\
\text { entrevistados }\end{array}$ & $\begin{array}{c}\text { Total } \\
\text { entrevistados }\end{array}$ \\
\hline Andes & Suroeste & 4 & 6 & 10 \\
\hline Angostura & Norte & 4 & 5 & 9 \\
\hline Apartadó & Urabá & 8 & 5 & 13 \\
\hline Caucasia & Bajo Cauca & 6 & 3 & 9 \\
\hline Dabeiba & Occidente & 8 & 3 & 11 \\
\hline Girardota & Valle de Aburrá & 7 & 6 & 13 \\
\hline Guatapé & Oriente & 12 & 3 & 15 \\
\hline Murindó & Urabá & 7 & 2 & 9 \\
\hline Nechí & Bajo Cauca & 7 & 4 & 11 \\
\hline Puerto Berrío & Magdalena Medio & 10 & 1 & 11 \\
\hline Remedios & Nordeste & 8 & 1 & 9 \\
\hline Rionegro & Oriente & 8 & 5 & 13 \\
\hline Sabaneta & Valle de Aburrá & 10 & 3 & 13 \\
\hline Santa Fe de Antioquia & Occidente & 11 & 5 & 16 \\
\hline Urrao & Suroeste & 6 & 6 & 12 \\
\hline Yarumal & Norte & 4 & 4 & 8 \\
\hline Yondó & Magdalena Medio & 6 & 5 & 11 \\
\hline \multicolumn{2}{|l|}{ Total entrevistados } & 126 & 67 & 193 \\
\hline
\end{tabular}

Fuente: elaboración propia. 
Posteriormente, se llevó a cabo el procesamiento de la información con base en las categorías previamente definidas a partir de la teoría y complementadas con las categorías emergentes que se evidenciaron en el análisis de las entrevistas. De esta forma se definieron cinco grandes categorías: a) la discrecionalidad y autonomía de los $\mathrm{BNC}$; b) la reconfiguración o elaboración final de políticas a partir de los BNC; c) los aspectos que (des) motivan el trabajo de los BNC; d) los controles y limitaciones del trabajo de nivel de calle; y e) el relacionamiento entre la ciudadanía y los BNC.

Las guías de entrevistas realizadas a funcionarios contaron con grupos de preguntas dirigidas a identificar el quehacer de los actores de la política, describir sus expectativas frente a lo que debería ser la PPSAN y calificar las dinámicas exógenas — características demográficas, geográficas, composición de las estructuras productivas, entre otros- y endógenas categoría del municipio, estructura de la administración municipal, nivel de relacionamiento de los actores, entre otros - que afectan las políticas públicas en el ámbito territorial con el fin de identificar patrones que pudieran influir en la implementación de la política y que no estuvieran directamente asociados al actuar de los BNC. Por su parte, las guías aplicadas a beneficiarios se enfocaron en detectar su relación con la administración municipal, además de su percepción sobre los bienes y servicios provistos por el gobierno. En este aspecto, las entrevistas permitieron señalar la relación entre los ciudadanos y BNC como punto importante de estudio.

Las entrevistas proporcionaron una visión de la realidad que construye cada individuo en un espacio (Guber, 2001), en el que se suministró información sobre el desarrollo de los programas y proyectos en materia de seguridad alimentaria y nutricional (SAN); además, se evidenció la forma en que interactúan los diferentes actores y cómo esto deriva en una política pública particular que puede distar de lo plasmado en lo documental y normativo. El volumen de las entrevistas realizadas permitió contrastar los diferentes relatos, las características constantes y las particularidades de los BNC en el PPSAN.

Se asumió entonces la perspectiva constructivista propuesta por Kathy Charmaz (2009). En ella, el conocimiento se presenta como una producción social donde la investigación es un proceso y un producto de 
condiciones preexistentes ligadas a factores territoriales que influyen en el investigador, los entrevistados y los resultados. Por consiguiente, para analizar la información obtenida en las entrevistas se emplearon técnicas afines con la teoría fundamentada para codificar la información y así llegar a explicaciones más plausibles de las acciones de los actores involucrados en el estudio.

Para ello se utilizó el método de codificación propuesto por Anselm Strauss y Juliet Corbin (2002). Inicialmente, las entrevistas se clasificaron en tres grandes grupos: burócratas, burócratas de nivel de calle y beneficiarios. De esta forma se observaron diferencias y similitudes en cada uno de los discursos según el rol del actor en la política pública. Posteriormente, se inició la codificación y sistematización de los datos mediante un examen detallado de cada una de las entrevistas. Esto permitió identificar los puntos de convergencia y divergencia, además de clasificarlos con códigos analíticos que expresan de manera sucinta lo dicho en la entrevista, encuadrándolos bajo los campos establecidos que responden al marco teórico del estudio. Con el fin de no limitar la información provista por cada entrevistado, se conformaron nuevas categorías que emergieron de los datos, facilitando así la comprensión del papel de los BNC en la implementación de las políticas públicas. Esto permitió el entendimiento de las particularidades de cada caso, además de los factores ausentes en la literatura estudiada.

Después de establecer las categorías, se procedió a realizar una comparación entre ellas usando los códigos extraídos de las entrevistas. Así se lograron identificar de manera clara los puntos en común y las divergencias entre cada categoría, evidenciando, a su vez, las particularidades territoriales y las diferentes dinámicas en materia de implementación de políticas públicas en los municipios del departamento de Antioquia. El análisis se realizó en una lógica territorial con el fin de identificar los contrastes entre las subregiones y preservar el anonimato de los funcionarios y ciudadanos que participaron en el estudio.

Finalmente, se construyeron textos analítico-descriptivos con base en estas categorías. Más adelante, se contrastaron entre sí para realizar un análisis correlacional de estos y se pudo evidenciar la relevancia de los BNC en la PPSAN en el departamento de Antioquia (véase gráfica 1). 
Gráfica 1. Proceso de codificación y análisis de información.

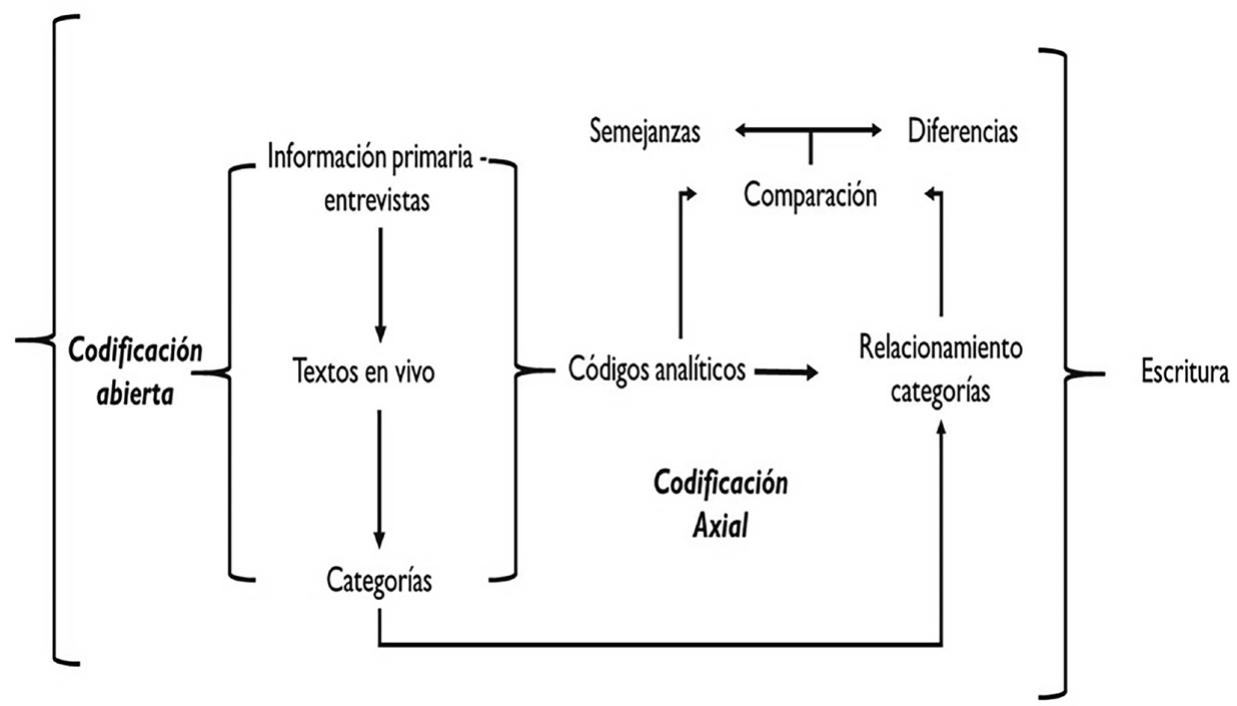

Fuente: elaboración propia.

\section{La discrecionalidad y autonomía de los burócratas de nivel de calle en la Política Pública de Seguridad Alimentaria y Nutricional}

La discrecionalidad y autonomía de los BNC se ven afectadas por factores externos que no pueden controlar, pero inciden directamente en las acciones que estos desarrollan día a día. Así, el análisis y codificación de las entrevistas permitió identificar factores que condicionan el adecuado desarrollo de las acciones en materia de PPSAN y que pueden enmarcarse en dos grandes campos: las condicionantes territoriales y las condiciones político-institucionales. Las tablas 2 y 3 muestran los principales factores asociados a cada uno de los campos, los territorios en donde se observaron, las problemáticas que generaban y su impacto en programas y proyectos específicos, o en la PPSAN en general.

Cada uno de estos factores tiene efectos en el uso de la discrecionalidad, pues funcionan como condicionantes del actuar de los BNC al limitar las acciones de la PPSAN, lo que lleva a adecuar los procesos a las características específicas de cada territorio. 
Tabla 2. Factores territoriales que afectan la Política Pública de Seguridad Alimentaria y Nutricional y la labor de los burócratas de nivel de calle.

\begin{tabular}{|c|c|c|c|}
\hline $\begin{array}{c}\text { Factores } \\
\text { Territoriales }\end{array}$ & $\begin{array}{c}\text { Territorios donde } \\
\text { se identificó el } \\
\text { factor }\end{array}$ & $\begin{array}{c}\text { Problemáticas asociadas } \\
\text { al factor }\end{array}$ & $\begin{array}{c}\text { Programa o proyecto } \\
\text { afectado }\end{array}$ \\
\hline $\begin{array}{l}\text { Dispersión } \\
\text { Territorial }\end{array}$ & $\begin{array}{l}\text { - Bajo Cauca } \\
\text { - Urabá } \\
\text { - Magdalena } \\
\text { Medio }\end{array}$ & $\begin{array}{l}\text { Dificultad en la distribución de } \\
\text { alimentos y en la prestación } \\
\text { de servicios por las distancias } \\
\text { de los asentamientos humanos } \\
\text { (problemas de movilidad terres- } \\
\text { tre y fluvial). }\end{array}$ & $\begin{array}{l}\text { - Programa de } \\
\text { Alimentación Escolar } \\
\text { (PAE). } \\
\text { - Proyectos } \\
\text { productivos. } \\
\text { - Proyecto de } \\
\text { paquetes alimentarios. }\end{array}$ \\
\hline \begin{tabular}{|l|} 
Configuración \\
de estructuras \\
productivas \\
agropecuarias
\end{tabular} & $\begin{array}{l}\text { - Oriente } \\
\text { - Occidente } \\
\text { - Norte } \\
\text { - Nordeste }\end{array}$ & $\begin{array}{l}\text { Baja producción de alimentos } \\
\text { en el ámbito municipal y difi- } \\
\text { cultades de autoabastecimiento } \\
\text { que no permiten una provisión } \\
\text { directa a los programas territo- } \\
\text { riales. Compra de alimentos a } \\
\text { otras subregiones y departamen- } \\
\text { tos que no permite el fortaleci- } \\
\text { miento de la producción local. }\end{array}$ & $\begin{array}{l}\text { - PAE. } \\
\text { - Centros de } \\
\text { Desarrollo Infantil } \\
\text { (CDI). }\end{array}$ \\
\hline $\begin{array}{l}\text { Utilización de } \\
\text { los suelos }\end{array}$ & $\begin{array}{l}\text { - Oriente } \\
\text { - Norte } \\
\text { - Valle de Aburrá } \\
\text { - Occidente } \\
\text { - Magdalena } \\
\text { Medio }\end{array}$ & $\begin{array}{l}\text { Desplazamientos de la produc- } \\
\text { ción agropecuaria por el turis- } \\
\text { mo, la urbanización y actividad } \\
\text { inmobiliaria, así como la con- } \\
\text { centración en único sector pro- } \\
\text { ductivo como la ganadería. }\end{array}$ & $\begin{array}{l}\text { - La Política Pública } \\
\text { de Seguridad } \\
\text { Alimentaria y } \\
\text { Nutricional en } \\
\text { general. }\end{array}$ \\
\hline
\end{tabular}

Fuente: elaboración propia.

Tabla 3. Factores político-institucionales que afectan la Política Pública de Seguridad Alimentaria y Nutricional y la labor de los burócratas de nivel de calle.

\begin{tabular}{|c|c|c|c|}
\hline $\begin{array}{l}\text { Factores político- } \\
\text { institucionales }\end{array}$ & $\begin{array}{c}\text { Territorios } \\
\text { donde se } \\
\text { identificó el } \\
\text { factor }\end{array}$ & $\begin{array}{c}\text { Problemáticas asociadas } \\
\text { al factor }\end{array}$ & $\begin{array}{c}\text { Programa o proyecto } \\
\text { afectado }\end{array}$ \\
\hline $\begin{array}{l}\text { Condiciones } \\
\text { físicas y } \\
\text { locativas para } \\
\text { la prestación de } \\
\text { servicios }\end{array}$ & $\begin{array}{l}\text { - Magdalena } \\
\text { - Medio } \\
\text { - Nordeste } \\
\text { - Norte } \\
\text { - Suroeste } \\
\text { - Urabá } \\
\text { - Bajo Cauca }\end{array}$ & $\begin{array}{l}\text { Dificultades en la prestación de } \\
\text { servicios de alimentación por } \\
\text { problemas de estructura física } \\
\text { y sanidad en instalaciones terri- } \\
\text { toriales. }\end{array}$ & $\begin{array}{l}\text { - Comedores Escolares. } \\
\text { - Comedores } \\
\text { Comunitarios. }\end{array}$ \\
\hline
\end{tabular}


Tabla 3. (Continuación)

\begin{tabular}{|c|c|c|c|}
\hline $\begin{array}{l}\text { Factores político- } \\
\text { institucionales }\end{array}$ & $\begin{array}{l}\text { Territorios } \\
\text { donde se } \\
\text { identificó el } \\
\text { factor }\end{array}$ & $\begin{array}{c}\text { Problemáticas asociadas } \\
\text { al factor }\end{array}$ & $\begin{array}{c}\text { Programa o proyecto } \\
\text { afectado }\end{array}$ \\
\hline $\begin{array}{l}\text { Idoneidad y } \\
\text { suficiencia de } \\
\text { funcionarios }\end{array}$ & $\begin{array}{l}\text { - Norte } \\
\text { - Magdalena } \\
\text { Medio } \\
\text { - Oriente }\end{array}$ & $\begin{array}{l}\text { Contratación de personal no } \\
\text { calificado que afectaba el ne- } \\
\text { gativamente el desarrollo de } \\
\text { las acciones de gobierno en los } \\
\text { territorios; así como la falta de } \\
\text { nutricionistas para la atención } \\
\text { adecuada de beneficiarios. }\end{array}$ & $\begin{array}{l}\text { - Proyectos } \\
\text { productivos. } \\
\text { - Centros de Desarrollo } \\
\text { Infantil (CDI). }\end{array}$ \\
\hline $\begin{array}{l}\text { Categoría de } \\
\text { la entidad } \\
\text { territorial }\end{array}$ & $\begin{array}{l}\text { - Municipios } \\
\text { de quinta y } \\
\text { sexta categoría } \\
\text { (véase tabla 1) }\end{array}$ & $\begin{array}{l}\text { Falta de recursos para alcanzar } \\
\text { coberturas deseadas. Limitacio- } \\
\text { nes en términos generales para } \\
\text { el desarrollo de la PPSAN. }\end{array}$ & $\begin{array}{l}\text { - La Política Pública de } \\
\text { Seguridad Alimentaria } \\
\text { y Nutricional en } \\
\text { general. }\end{array}$ \\
\hline $\begin{array}{l}\text { Desembolso de } \\
\text { recursos }\end{array}$ & $\begin{array}{l}\text { - Urabá } \\
\text { - Bajo Cauca } \\
\text { - Occidente }\end{array}$ & $\begin{array}{l}\text { Problemas de articulación lo- } \\
\text { cal-regional para el desembol- } \\
\text { so de recursos dirigidos a pro- } \\
\text { gramas de la PPSAN causando } \\
\text { interrupción de estos. }\end{array}$ & $\begin{array}{l}\text { - Programa de } \\
\text { Alimentación Escolar } \\
\text { (PAE). } \\
\text { - Paquetes } \\
\text { alimentarios. }\end{array}$ \\
\hline $\begin{array}{l}\text { Planeación de } \\
\text { los procesos de } \\
\text { contratación }\end{array}$ & Todos & $\begin{array}{l}\text { Interrupción de prestación de } \\
\text { servicios y entrega de vienes } \\
\text { por retrasos en los procesos de } \\
\text { contratación de terceros priva- } \\
\text { dos, esto genera discontinui- } \\
\text { dad en los programas y proyec- } \\
\text { tos territoriales. }\end{array}$ & $\begin{array}{l}\text { - La Política Pública de } \\
\text { Seguridad Alimentaria } \\
\text { y Nutricional en } \\
\text { general. }\end{array}$ \\
\hline $\begin{array}{l}\text { Entidad } \\
\text { encargada } \\
\text { de la PPSAN } \\
\text { (ubicación } \\
\text { sectorial de la } \\
\text { política) }\end{array}$ & Todos & $\begin{array}{l}\text { Implementación de la PPSAN } \\
\text { de acuerdo a las orientaciones } \\
\text { de la entidad encargada (salud, } \\
\text { educación, agricultura, bien- } \\
\text { estar social). Acciones enmar- } \\
\text { cadas en sectores de política } \\
\text { que establecen un foco de ac- } \\
\text { tuación de acuerdo a la entidad } \\
\text { encargada, limitando un desa- } \\
\text { rrollo integral de la política. }\end{array}$ & $\begin{array}{l}\text { - La Política Pública de } \\
\text { Seguridad Alimentaria } \\
\text { y Nutricional en } \\
\text { general. }\end{array}$ \\
\hline \begin{tabular}{|l} 
Disputas \\
político- \\
electorales
\end{tabular} & $\begin{array}{l}\text { - Urabá } \\
\text { - Oriente } \\
\text { - Magdalena } \\
\text { Medio }\end{array}$ & $\begin{array}{l}\text { Problemas de articulación y } \\
\text { coordinación interinstitucional } \\
\text { por disputas entre actores } \\
\text { políticos y burócrata; además, } \\
\text { establecimiento de clientelas } \\
\text { políticas en el marco de } \\
\text { proyectos de la PPSAN. }\end{array}$ & $\begin{array}{l}\text { - Paquetes } \\
\text { alimentarios. }\end{array}$ \\
\hline
\end{tabular}


Tabla 3. (Continuación)

\begin{tabular}{|l|l|l|l|}
\hline $\begin{array}{c}\text { Factores político- } \\
\text { institucionales }\end{array}$ & $\begin{array}{c}\text { Territorios } \\
\text { donde se } \\
\text { identificó el } \\
\text { factor }\end{array}$ & $\begin{array}{c}\text { Problemáticas asociadas } \\
\text { al factor }\end{array}$ & $\begin{array}{c}\text { Programa o proyecto } \\
\text { afectado }\end{array}$ \\
\hline $\begin{array}{l}\text { Hábitos y } \\
\text { prácticas } \\
\text { alimentarias }\end{array}$ & $\begin{array}{l}\text { - Urabá } \\
\text { - Occidente } \\
\text { - Bajo Cauca }\end{array}$ & $\begin{array}{l}\text { Establecimiento de minutas } \\
\text { alimenticias que no } \\
\text { corresponden a los hábitos y } \\
\text { prácticas alimentarias de los } \\
\text { territorios }\end{array}$ & $\begin{array}{l}\text { - Programa de } \\
\text { Alimentación Escolar }\end{array}$ \\
\hline
\end{tabular}

Fuente: elaboración propia.

Además de esto, se debe tener en cuenta que la discrecionalidad varía según el tipo de bien y servicio provisto, pues la estructura de algunos programas y proyectos es más rígida. Se pueden tomar como referencia los casos del Programa de Alimentación Escolar (PAE) y los Centros de Desarrollo Infantil (CDI), pues cuentan con una normatividad clara, una estructura de trabajo definida, un público objetivo específico y unos mecanismos de control impuestos por la nación, disminuyendo así la discrecionalidad y limitando las interpretaciones de la norma.

En el caso de estos dos programas, los beneficiarios hacen parte de una población de especial protección —niños, niñas y adolescentes (NNA) , lo cual funciona a favor de su rigidez e incentiva a los BNC a seguir los lineamientos preestablecidos con el fin proveer bienes y servicios de alta calidad. En todas las entrevistas referidas al PAE y a los CDI los participantes identificaron las bondades de ambos programas y sus beneficios sobre los NNA, por lo que su desempeño se direccionó a seguir los lineamientos establecidos, haciendo lo estrictamente necesario para alcanzar los objetivos propuestos y tener un efecto positivo en los beneficiarios.

Así, estos factores desencadenan un comportamiento en doble vía. Por un lado, los BNC hacen lo que deben hacer dada la rigidez normativa y su motivación por prestar un buen servicio acorde con la importancia del grupo poblacional atendido. Por otro lado, hacen lo que pueden hacer bajo las condiciones desfavorables que se presentan en el desarrollo del programa que implementan. Por lo tanto, se encontró que la vinculación de ambos programas a ámbitos superiores de gobierno implica una estructura más rígida y controlada, disminuyendo la discrecionalidad. Sin embargo, se debe 
considerar que los planteamientos teóricos sugieren que el aumento de control resulta, a su vez, en una mayor cantidad de procesos burocráticos, siendo este uno de los principales factores que genera brechas de implementación. Por ejemplo, aunque se reconoce la discrecionalidad para darle un enfoque a los programas, las acciones se limitan a cumplir los lineamientos, como lo expresa uno de los BNC: «Acá todos los programas tienen su enfoque, cada persona que lo está manejando lo direcciona a ese enfoque, por ejemplo, yo en alimentación escolar, yo no puedo meter al que yo quiera, ino!, hay una plataforma donde están los niños que deben de ser beneficiados con el programa» (Burócrata PB5, comunicación personal, octubre 5, 2018).

Otros BNC de esta política tienen condiciones diferentes. Algunos de los proyectos que no se enmarcan en dinámicas del ámbito nacional cuentan con mayor flexibilidad, lo que incentiva la discrecionalidad de los BNC. Los proyectos productivos, las huertas caseras, las huertas escolares, los paquetes alimentarios, la asesoría técnica agropecuaria y otros proyectos asociados con la formación en hábitos alimentarios saludables cuentan con un grupo de profesionales que, si bien desarrollan su trabajo partiendo de las directrices exigidas por estos proyectos, también los modifican con el fin de generar, desde su perspectiva, un mayor impacto.

En el caso de las acciones asociadas a proyectos productivos, huertas caseras y escolares, y paquetes alimentarios en Oriente, Occidente, Norte, Suroeste y Urabá, los participantes afirmaron haber realizado modificaciones con el fin de incluir beneficiarios que no cumplían con los requisitos establecidos, pero que a su criterio tenían mayores necesidades que otros ciudadanos que eran aptos. Pese a ser excepciones, esto refuerza la idea de discrecionalidad inherente al BNC, que puede disponer de ella siempre que lo considere necesario, evidenciando así su autonomía. Estos actores crean estructuras de trabajo y marcos en los que pueden clasificar a los ciudadanos como aptos o no aptos para recibir la ayuda gubernamental:

Uno de los principales problemas ha sido el puntaje del Sisbén, ${ }^{1}$ porque aquí las personas están por encima de 51 puntos, por ejemplo, y para ingresar al programa deben estar en 50, entonces nosotros

\footnotetext{
${ }^{1}$ El Sistema de Identificación de Potenciales Beneficiarios de Programas Sociales (Sisbén) clasifica a través de un puntaje a la población de acuerdo con sus condiciones socioeconómicas. Es utilizado para identificar de manera rápida y objetiva a la población en situación de pobreza y vulnerabilidad para focalizar la inversión social y garantizar que esta sea asignada a quienes más lo necesitan.
} 
hemos tenido que jugar con eso, buscar formas más adecuadas de priorizar, al menos en lo local, porque lo departamental toca hacer un oficio para que la gobernación permita la inclusión de esas personas que no están dentro del puntaje, entonces qué hacemos, investigamos y uno más o menos sabe quién si necesita las ayudas y así resulta más bueno el programa, porque llega a quien lo necesita (Burócrata GP3, comunicación personal, octubre 22, 2018).

En otros casos, la discrecionalidad se encuentra mediada por factores como los que contienen las tablas 2 y 3 . Por ejemplo, el proyecto de paquetes alimentarios que consiste en la entrega de alimentos no perecederos a personas en situación de pobreza es utilizado por actores políticos para ampliar su caudal electoral, lo que condiciona su implementación y el accionar de los BNC. Esto implica que otros actores con poder en la comunidad y el territorio aprovechen la discrecionalidad para beneficios particulares, es decir, a diferencia de los planteamientos de Lipsky (2010), en los cuales es utilizada para satisfacer deseos particulares del BNC o su organización, esta pasa a ser objeto de disputa entre los actores políticos para fines electorales, pasando de la capacidad de juicio del BNC a la violación de la norma expresada por Michael Hill (1984):

Los paquetes alimentarios se convirtieron en unas cuotas políticas. Cuando ingresamos al trabajo en esta administración nos tocaba hacer la liquidación del proyecto en el año anterior y uno debe manejar un registro, una planilla que nos manda el departamento donde la persona firma y huella [sic] de los que hayan recibido el complemento, encontramos que no había un registro sino una lista, entonces empezamos a buscar de esa lista a las personas y resulta que se envió la planilla con unas personas que eran niveles 1 y 2 y los paquetes no fueron entregados a niveles 1 y 2 , simplemente fueron una fachada; entonces encontramos que cuando se entregaban los paquetes la condición era, votar por esa persona, [...] nos tocó buscar 180 personas que estaban en una lista ficticia y que no fueron beneficiadas (Burócrata G9, comunicación personal, octubre 24, 2018).

Desde esta perspectiva, la discrecionalidad de los BNC es instrumentalizada y utilizada para cumplir promesas electorales referidas a la inclusión de beneficiarios en programas sin tener en consideración los criterios preestablecidos, obligando a los BNC a responder a demandas externas a sus deseos y a los de su organización. Esto perjudica a un potencial público 
objetivo con mayores necesidades que aquellos que deben incluirse en el programa por factores políticos.

En otros casos donde se observó el factor relacionado con la subcontratación, se pudo evidenciar que los BNC que se encontraban motivados y comprometidos con los objetivos de la política debían realizar un uso mayor de su discrecionalidad para menguar la incapacidad de los BNC asignados por favoritismo y así entregar los bienes y servicios de manera adecuada a la comunidad. Por ejemplo, en el caso de las asesorías técnicas y la formación en hábitos de alimentación saludable, algunos de los BNC afirmaron tener que realizar acciones adicionales a las preestablecidas, dado que sus compañeros de equipo realizaban un trabajo precario y, en ocasiones, en detrimento del objeto del proyecto:

Los alcaldes llegan a una administración municipal con unos favores políticos y esos favores políticos desfavorecen a los programas, ¿en qué sentido? Contratan personas que no cumplen con la experiencia y ellos, por política, envían a cualquier persona, eso hace que las personas que ya venían en el programa con una formación, una capacitación, se queden relegadas porque de pronto no fueron del grupo político o no estuvieron con él y el programa empieza a perder calidad por ese lado [...], y le toca a uno continuar un proceso a uñas, porque no hay más nada qué hacer con esas personas y eso va en desmejora de la calidad del programa (Burócrata PB6, comunicación personal, julio 10, 2019).

En lo que respecta a la asesoría técnica agropecuaria y la formación en hábitos alimenticios saludables, la discrecionalidad es mucho mayor. En primer lugar, los BNC encargados no tienen mecanismos de control más allá de planillas de asistencia que solo garantizan la visita o la asesoría. Sin embargo, esta discrecionalidad no tiene una carga valorativa per se, y en el caso particular favorece al desarrollo de las acciones, permitiéndole a los BNC ir más allá de lo establecido. En algunos casos, los funcionarios imparten charlas no estipuladas o desarrolladas en otros periodos de gobierno que pueden tener efectos positivos en los hábitos alimenticios de las comunidades. En otros, se brinda una asesoría técnica con un acompañamiento particularizado e integral, superando la mera enseñanza de condiciones generales para el desarrollo del programa que debe realizar el BNC. 
Desde la perspectiva de los BNC, estas funciones adicionales deben realizarse debido a la limitación de los lineamientos de las políticas, que no responden a las necesidades de las comunidades, puesto que estas requieren de un acompañamiento más integral que garantice la perdurabilidad y sostenibilidad de los proyectos. Algunos BNC afirman que uno de los principales factores que afectan la perdurabilidad del proyecto es el acompañamiento precario y altamente técnico sin componente social, además de la inestabilidad laboral de los BNC, que por lo general cuentan con una vinculación no mayor a un año, repercutiendo así en los procesos de la PPSAN:

Para mí, realmente, la principal limitante es la manera como se crean los lineamientos técnicos y administrativos, es una opinión personal, y es la manera como desde Bogotá se imaginan un programa de una manera tan estándar para sitios tan específicos, es [...] tratar de encasillar un montón de componentes nutricionales, técnicos, sociales y pensar que ...] va a ser una herramienta que va a servir para todo el país cuando hay territorios tan específicos, porque muchas veces se desconoce la tradición, la tradición de cómo se alimentan las comunidades (Burócrata SF12, comunicación personal, noviembre 8,2018 ).

[144] En general, se logró identificar que el uso de la discrecionalidad se ciñe a momentos de necesidad o presión, y que esta se utiliza para obtener retribuciones simbólicas de carácter social y moral, es decir, a excepción del caso de los paquetes alimentarios, esta representa una fuente de satisfacción para los BNC, pues los lleva a pensar que están haciendo lo correcto en beneficio de la población que lo requiere. De igual manera, en los casos en los que la discrecionalidad es menor, la decisión de apegarse a las reglas pasa por un factor moral y social de suma importancia para el grupo poblacional atendido - $\operatorname{los}$ NNA—.

\section{Burócratas de nivel de calle y ciudadanos: la elaboración final de las políticas}

La elaboración final o reformulación de políticas se refiere concretamente a los cambios realizados en la política durante su proceso de implementación. En este aspecto, los BNC son los elaboradores finales, pues son quienes ejecutan las decisiones tomadas en ámbitos superiores de gobierno en realidades específicas que están permeadas por factores territoriales — políticos, sociales y económicos-, institucionales, organizacionales y personales, dando cabida a una brecha entre lo formulado y lo implementado. Una posición activa 
o pasiva conlleva a que el BNC de la PPSAN se comporte de una manera distinta. Si bien Lipsky (2010) anota que los beneficiarios tienden a tomar un rol pasivo y evitan la confrontación con el BNC para evadir las posibles represalias que puedan tomarse contra ellos, en el caso de la PPSAN los ciudadanos se comportan de diferentes maneras.

El comportamiento más común en las entrevistas a funcionarios y beneficiarios es el pasivo: son receptores de ayudas gubernamentales y no tienen ningún tipo de objeción sobre la forma y el contenido de lo que provee el Estado. Este tipo de ciudadanos toman una postura de sumisión, lo que permite que el BNC establezca una relación de jerarquía y mando que determina las condiciones para la prestación del servicio o la entrega del bien. Este tipo de beneficiarios, que solo mencionaron la importancia de las acciones y la necesidad de recibir más beneficios, estuvo presente en cada uno de los proyectos identificados que se enmarcan en la PPSAN.

Frente a este tipo de conducta y la posición que toman los BNC al respecto, es posible identificar algunas fallas que condicionan su comportamiento y el de sus organizaciones, transformado a su vez la PPSAN. La posición de sumisión genera un aumento de acciones de corte asistencial que establecen una relación de patronazgo entre políticos-burócratas y ciudadanía. Incluso los BNC reconocen que estas no tienen ningún impacto en el tiempo, pues no buscan romper con las causas de problemáticas como la inseguridad alimentaria, la desnutrición y los inadecuados hábitos alimenticios.

Un segundo tipo de comportamiento se puede asociar con una conducta emprendedora por parte de los beneficiarios. Este tipo se circunscribe especialmente a acciones como los proyectos productivos, las huertas caseras y los mercados campesinos. Aquí la ciudadanía asume un papel más activo y busca mejorar las condiciones de los programas existentes, además de llevar a cabo acciones individuales que permitan reforzar los proyectos emprendidos por el gobierno local y departamental para que sean viables y se les dé mayor continuidad, lo que proporciona mejores resultados según los mismos beneficiarios y BNC.

Esto hace que los BNC de la PPSAN tomen una actitud proactiva y además realicen acciones adicionales como retribución a la conducta del beneficiario. No se establece una relación de jerarquía, sino de cooperación entre las partes, abriendo los canales de comunicación que incentivan a los BNC a satisfacer las necesidades de los ciudadanos. Para ello, hacen uso de su 
discrecionalidad y brindan un acompañamiento mayor, además, flexibilizan los requerimientos para que los ciudadanos tengan acceso a otros beneficios e inciden en el gobierno para llevar a cabo modificaciones basadas en su experiencia y relación con la ciudadanía. Por ejemplo, en el caso de proyectos productivos se encontraron testimonios en los que los beneficiarios y los BNC afirmaban tener un acompañamiento más integral que no se limitaba a su labor específica de manera reiterativa:

A nosotros la Alcaldía desde la Secretaría nos entregan unas cositas, insumos y acompañamiento técnico, pero eso, la verdad, no es suficiente. Nosotros tenemos que tener [sic] otros cultivos y nuestro ganadito, cierto, entonces nosotros trabajamos muy de cerquita con los muchachos de la Secretaría que nos hacen el acompañamiento y ellos nos ayudan y nos dan asesorías también para las otras cosas propias que nosotros hacemos aquí en la vereda (Beneficiario P6, comunicación personal, junio 18, 2019).

El tercer tipo de conducta que se pudo identificar es la de aquellos ciudadanos que toman una actitud de disgusto en la relación que establecen con el BNC. Se identificó en individuos que cuentan con apoyo político o que en épocas electorales fueron influenciados para votar por un determinado candidato con la promesa de ser incluidos en proyectos como paquetes alimentarios. Esta conducta es la más problemática y puede hacer que la discrecionalidad vaya en contra de los lineamientos de política, perjudicando a otros posibles beneficiarios.

En este caso, el BNC no solo se enfrenta a la ciudadanía, sino también a actores políticos que ejercen presión para incluir a sus electores en el proyecto en cuestión. Frente a esta situación, el BNC debe darles prioridad a aquellos individuos propuestos por el actor político, siempre y cuando cumplan con los requisitos para ser incluidos en el programa. Además, deben flexibilizar ciertos requerimientos para aquellos que no cumplan con todas las condiciones. Aquí la discrecionalidad es utilizada por un tercero, generando una brecha y excluyendo posibles beneficiarios que necesiten más el apoyo gubernamental; además, se establece una relación clientelar a partir de la PPSAN. Tal situación ha llevado a que los BNC incluso sean amenazados por los beneficiarios que tienen respaldo de actores políticos en Oriente y Valle de Aburrá. Por ejemplo, los adultos mayores que no recibieron el paquete alimentario prometido hicieron sus reclamos a través de los concejales después de no verse beneficiados por el programa: 
Entonces empezaron a utilizar a los concejales y empezaron a ir al concejo, «iEs que yo vote por ustedes!» y entonces el señor concejal venía con la potestad de indicar qué persona tenía que recibir el paquete [...] me han mandado hasta cartas de amenazas con las abuelas porque no les he entregado un paquete alimentario (Funcionario G15, comunicación personal, octubre 24, 2018).

Con respecto a otros programas como el PAE o los CDI, no se observaron conductas particulares de los beneficiarios o los BNC, y en general se llevan a cabo procedimientos altamente estandarizados. En este sentido, las fallas, brechas y resultados finales del programa no dependen del BNC directamente, sino de elementos territoriales y factores externos que limitan su adecuado desarrollo.

Por consiguiente, se presenta una relación entre el ámbito del que provienen las acciones y el grado de discrecionalidad del que dispone el $\mathrm{BNC}$, pues es en las acciones del ámbito territorial donde intervienen el gobierno municipal $y$, en ocasiones, el departamental. En este último se hace mayor uso de la discrecionalidad, aunque esto no implica que sea significativa, pues en la mayoría de los casos se pudo evidenciar que los BNC siguen los procedimientos establecidos y la discrecionalidad se da en razón de su relación con los beneficiarios y los factores territoriales y externos que condicionan su accionar.

\section{Consideraciones finales}

Aunque el análisis en el ámbito de BNC en la PPSAN no explica las brechas que se presentan en la política en su totalidad, sí aporta a la comprensión de estas. Algunas variables como la capacidad económica, la robustez institucional, la dispersión del territorio, entre otras, parecen ser más determinantes al momento de explicar el resultado final de la PPSAN. Si bien los BNC como implementadores tienen la capacidad de transformar la política, el uso de su discrecionalidad se limita a casos muy particulares y tiene como fin mejorar las condiciones de los beneficiarios y, por ende, de la política.

La discrecionalidad y autonomía de los BNC lleva a que las políticas públicas tomen una forma particular al momento de implementarse, alejándose de lo planteado en los documentos normativos y técnicos que las aprueban. Desde esta perspectiva, las políticas públicas no son, en ningún 
caso, un documento, sino el conjunto de decisiones y acciones que se llevan a cabo en los territorios y que tienen efecto en un conjunto de personas.

En términos generales, el accionar de los BNC se puede enmarcar en dos formas de proceder: en la primera, hacen lo que deben, siguen claramente los lineamientos establecidos por los ámbitos superiores de gobierno — caso PAE y $\mathrm{CDI}$ - para desarrollar su labor diaria; en la segunda intervienen los condicionantes y factores territoriales y externos, llevando a los BNC a hacer lo que pueden, tal es el caso del PAE, los CDI y los demás proyectos de orden departamental y municipal. Los BNC deben trabajar bajo unas condiciones que no son óptimas. La falta de recursos económicos, las presiones políticas, el comportamiento de la ciudadanía y la ausencia de lineamientos acordes a las necesidades territoriales restringen su capacidad para implementar adecuadamente las acciones que tienen a cargo.

La discrecionalidad varía según el tipo de programa implementado. Aunque las acciones que se enmarcan en la PPSAN son diversas, es posible hablar de dos grandes bloques: en el primero están las provenientes del ámbito nacional, como los CDI y el PAE, que están a cargo de representantes de este ámbito de gobierno — Instituto Colombiano de Bienestar Familiar

[148] (ICBF) y Ministerio de Educación-. Por consiguiente, su estructura es mucho más rígida, tiene una normatividad más definida con mecanismos de control establecidos, reduciendo así la capacidad discrecional de los BNC, lo que desfavorece las modificaciones en la política desde este ámbito. Sin embargo, la política se ve afectada en función de variables que no pueden ser controladas por los $\mathrm{BNC}$, es decir, esta cambia con respecto a su planteamiento original, pero no a causa de los implementadores.

El segundo bloque está compuesto por los programas propios de los municipios o que tienen acompañamiento del departamento. Estos suelen ser menos rígidos y son controlados por los mismos actores municipales, lo que favorece el uso de la discrecionalidad de los BNC, beneficiando, en su mayoría, a los ciudadanos. En este sentido, los BNC mejoran y potencian la política, bien sea realizando acciones adicionales para lograr mejores resultados o modificando los requerimientos para beneficiar a la población que más lo necesita.

Por otro lado, el clientelismo y la subcontratación se deben considerar como fenómenos estrechamente relacionados que inciden directamente sobre 
el accionar de los BNC. Los actores políticos aprovechan la discrecionalidad de los implementadores para consolidar su caudal electoral, lo que impide que los BNC desarrollen de manera adecuada su trabajo, llevándolos a transgredir las normas. Asimismo, la contratación de los BNC por favoritismo político sobrecarga a los BNC que se encuentran en la política por sus capacidades y conocimientos, y aumenta el uso de su discrecionalidad para subsanar el vacío dejado por sus pares.

Por consiguiente, el clientelismo aumenta el uso de la discrecionalidad de los BNC para fines que no están relacionados con la política, lo que obliga a los implementadores a responder a factores que no hacen parte de sus deseos ni los de la organización. Así, su discrecionalidad es utilizada por actores políticos para cumplir promesas electorales de inclusión de beneficiarios a programas que inicialmente no se encuentran en su ámbito de control, pero que gracias a su poder en el ámbito territorial logran ejercer la presión necesaria sobre los BNC para beneficiar a sus electores.

Otro factor significativo para el cambio de facto de la PPSAN y el uso de la autonomía y discrecionalidad es el comportamiento de los ciudadanos y beneficiarios. La actitud pasiva, emprendedora o de disgusto influencia el accionar del BNC, que en el primer caso se limita a hacer lo que debe, en el segundo utiliza su discrecionalidad para ayudar más a los participantes de los proyectos y en el tercero se ve obligado a violar la norma para beneficiar a la población que no se encuentra dentro del público objetivo del proyecto.

Los BNC al interior de la PPSAN representan un punto sustancial de investigación para comprender las dinámicas particulares de las acciones en los municipios del departamento de Antioquia. Sin embargo, la aplicación de la investigación a otros actores que no tienen contacto directo con la ciudadanía, pero que inciden sobre la labor llevada a cabo por los BNC, podría brindar una visión más amplia con respecto a la forma particular que toman las políticas públicas en el ámbito territorial, logrando identificar las brechas más significativas para darles tratamiento a partir de alternativas de solución viables y acordes con las necesidades y capacidades locales.

\section{Referencias bibliográficas}

1. Aguilar Villanueva, Luis F. (1992). El estudio de las políticas públicas. Introducción a la Biología Celular y Molecular. http://ibcm.blog.unq.edu.ar/wpcontent/uploads/sites/28/2014/03/Aguilar_Villanueva_Estudio-de-las-PP.pdf 
2. Arretche, Marta Tereza da Silva. (2001). Uma contribuição para fazermos avaliações menos ingênuas. En: Barreira, María Cecília Roxo Nobre e Brant, María do Carmo (orgs.). Tendências e Perspectivas na Avaliação de Políticas e Programas Sociais (pp. 43-56). São Paulo: Cenpec.

3. Charmaz, Kathy (2009). Shifting the Grounds: Constructivist Grounded Theory Methods for the Twenty-first Century. In: Morse, Janice; Stern, Phyllis; Corbin, Juliet; Bowers, Barbara; Charmaz, Kathy \& Clarke, Adele. Developing Grounded Theory: The Second Generation (pp. 127-154). Walnut Creek: Left Coast.

4. Cohen, Nissim \& Klenk, Tanja. (2019). Policy Re-Desing from the Street Level. In: Hupe, Peter (Ed.). Research Handbook on Street-Level Bureaucracy. The Ground Floor of Government in Context (pp. 209-222). Cheltenham: Edward Elgar. https://doi. org/10.4337/9781786437631.00024

5. Dussage, Mauricio; Cejudo, Guillermo y Pardo, María del Carmen (eds.). (2018). Estudio introductorio. En: Las burocracias a nivel de calle. Una ontología (pp. 9-26). México, D. F.: CIDE.

6. Espinoza, Henry y Huita, Franklin. (2012). Aplicación de incentivos en el sector público. Documento de Gestión Presupuestaria. Ministerio de Economía y Finanzas. https://www.mef.gob.pe/contenidos/presu_publ/estudios/DGP_inventivos_ fusionado.pdf

7. Evans, Tony. (2011). Professionals, Managers and Discretion: Critiquing StreetLevel Bureaucracy. The British Journal of Social Work, 41 (2), pp. 368-386. https://doi.

[150] org/10.1093/bjsw/bcq074

8. Gofen, Anat. (2013). Mind the Gap: Dimensions and Influence of Street-Level Divergence. Journal of Public Administration Research and Theory, 24, pp. 473-493. https://doi.org/10.1093/jopart/mut037

9. Guber, Rosana. (2001). La etnografía. Método, campo y reflexividad. Bogotá, D. C.: Norma.

10. Hill, Heather C. (2018). Comprender la implementación. Los recursos de los burócratas de nivel de calle para reformar. En: Dussage, Mauricio; Cejudo, Guillermo y Pardo, María del Carmen (eds.). Las burocracias a nivel de calle. Una ontología (pp. 127-162). México. D. F: CIDE.

11. Hill, Michael. (1984). Policy Process In The Modern State. Brighton: Wheatsheah.

12. Hupe, Peter (Ed.). (2019). Street-Level Bureaucracy as a Scholarly Theme. In: Research Handbook on Street-Level Bureaucracy. The Ground Floor of Government in Context (pp. 2-48). Cheltenham: Edward Elgar. https://doi. org/10.4337/9781786437631.00010

13. Jolly, Jean-François. (2007). Gobernancia de los territorios y gobierno del territorio en Colombia: El caso de la política pública de vivienda de interés social en Bogotá y Chiquinquirá. Papel Político, 11 (1), pp. 417-458. 
14. Kaufman, Herbert. (1973). Administrative Feedback; Monitoring Subordinates' Behavior. Washington, D. C: Brookings Inst.

15. Knoepfel, Peter; Larrue, Corinne; Varone, Frédéric e Hinojosa, Miriam. (2007). Hacia un modelo de análisis de políticas públicas operativo. Un enfoque basado en los actores, sus recursos y las instituciones. Ciencia Política, 2 (3), pp. 6-29.

16. Lipsky, Michael. (1969). Toward a Theory of Street-Level Bureaucracy. Institute for Research on Poverty Discussion Papers, pp. 48-69.

17. Lipsky, Michael. (2010). Street-Level Bureaucracy: Dilemmas of the Individual in Public Service. New York: Russell Sage Foundation.

18. Lipsky, Michael. (2018). Dilemas del individuo en el servicio público. En: Dussage, Mauricio; Cejudo, Guillermo y Pardo, María del Carmen (eds.). Las burocracias a nivel de calle. Una ontología (pp. 27-40). México, D. F.: CIDE.

19. MANÁ, Gerencia de Seguridad Alimentaria y Nutricional., Gobernación de Antioquia y Universidad de Antioquia. (2020). Plan Docenal de Seguridad Alimentaria y Nutricional 2020-2031. Medellín, Universidad de Antioquia.

20. Maynard-Moody, Steven y Musheno, Michael. (2018). Agente del Estado o agente de los ciudadanos: Dos narrativas sobre la discrecionalidad. En: Dussage, Mauricio; Cejudo, Guillermo y Pardo, María del Carmen (eds.). Las burocracias a nivel de calle. Una ontología (pp. 85-126). México, D. F.: CIDE.

21. Maynard-Moody, Steven y Portillo, Shannon. (2018). Teoría de la burocracia de nivel de calle. En: Dussage, Mauricio; Cejudo, Guillermo y Pardo, María del Carmen (eds.). Las burocracias a nivel de calle. Una ontología (pp. 41-84). México, D. F.: CIDE.

22. Mayntz, Renate. (1987). Sociología de la organización. Madrid: Alianza.

23. Medellín, Pedro. (2004). La política de las políticas públicas: propuesta teórica y metodológica para el estudio de las políticas públicas en países de frágil institucionalidad. Santiago de Chile: CEPAL.

24. Menon, Shaveta. (2018). Street-Level Bureaucracy in Tobacco Control: A Qualitative Study of Health Department in District Jalandhar, Punjab. Indian Journal of Community Health, 43 (4), pp. 270-273.

25. Mintzberg, Henry. (1984). La estructuración de las organizaciones. Barcelona: Ariel.

26. Navarrete Yáñez, Bernardo y Figueroa Rubio, Pamela. (2019). Los problemas de implementación top-down a nivel local. Un estudio de caso sobre seguridad ciudadana. Documentos y Aportes en Administración Pública y Gestión Estatal, 13 (20), pp. 81-109. https://doi.org/10.14409/da.v1i20.1293

27. Pressman, Jeffrey \& Wildavsky, Aaron. (1984). Implementation. How Great Expectations in Washington Are Dashed in Oakland; Or, Why It's Amazing that Federal Programs Work at All, This Being a Saga of the Economic Development Administration as Told by Two Sympathetic Observers Who Seek to Build Morals. Berkeley: University of California. 
28. Roth Duebel, André-Noël. (2018). Políticas públicas: formulación, implementación y evaluación. Bogotá, D. C.: Aurora.

29. Sevä, Mikael. (2015). The Decisive Role of Street-Level Bureaucrats in Environmental Management. (Doctoral Thesis). Luleå University of Technology, Luleå.

30. Strauss, Anselm y Corbin, Juliet. (2002). Codificación abierta. En: Bases de la investigación cualitativa. Técnicas y procedimientos para desarrollar la teoría fundamentada (pp. 110-132). Medellín: Universidad de Antioquia.

31. Weatherley, Richard \& Lipsky, Michael. (1977). Street-Level Bureaucrats and Institutional Innovation: Implementing Special Education Reform. Harvard Educational Review, 47 (2), pp. 171-197. https://doi.org/10.17763/haer.47.2.v870r1v16786270x

32. Weber, Max. (1993). Economía y sociedad. México, D. F.: Fondo de Cultura Económica.

33. Whitford, Aandrew. (2002). Decentralization and Political Control of the Bureaucracy. Journal of Theoretical Politics, 14 (2), pp. 167-193. https://doi. org/10.1177/095169280201400202

34. Williams, Rod. (2014). Examining the Role of Street-level Bureaucrats in the Implementation of an Affordable Rental Housing Policy for Extremely Low Income Households in the District of Columbia. (Theses Dissertation). University of Arkansas, Fayetteville.

35. Wright Mills, Charles. (1993). La elite del poder. México, D. F.: Fondo de Cultura Económica.

[152 ] 36. Zahariadis, Nikolaos. (2010). El marco de las corrientes múltiples. Estructura, limitaciones, perspectivas. En: Sabatier, Paul (ed.). Teoría del proceso de las políticas públicas (pp. 67-94). Buenos Aires: Westview. 